\title{
Caught in the act
}

Macrophage infiltration after nerve injury and in certain neurological diseases is closely related to pathogenic and restorative processes. So, an important problem in the field is to understand the kinetics of infiltration. In a recent paper, Bendszus and Stoll report on a method to monitor macrophage migration into injury sites in vivo using magnetic resonance imaging (MRI).

The method depends on the injection of superparamagnetic iron oxide particles, which are routinely used in people to diagnose liver tumours. As macrophages take up these particles, the local accumulation of 'loaded' cells $24 \mathrm{~h}$ after injection of the particles results in alterations of the MRI signal from the surrounding areas, owing to the strong paramagnetic effect.

Using this approach, the authors discovered that crushing the sciatic nerve was followed by a loss of the local MRI signal 1-8 days later. This loss was transient and correlated with the immunocytochemical detection of macrophages in the lesion site, providing support for the validity of the method. In addition, by manipulating the interval between particle injection and detection, Bendszus and Stoll found evidence that macrophages did not move together with the receding stump of the degenerating nerve, but remained stationary after invading the lesion site.

Last, the method can also differentiate between macrophage and microglial responses. As the two cell populations undergo phagocytic transformation and express similar markers after activation, it is difficult to tell them apart in tissue sections. Bendszus and Stoll observed that crushing the optic nerve, a site in which there is no macrophage infiltration but a strong microglial response, did not result in the local accumulation of the particles. This ability to differentiate between the two cell types might be useful in research and perhaps in the clinic.

Juan Carlos López

\section{(2) References and links}

ORIGINAL RESEARCH PAPER Bendszus, M. \& Stoll, G.

Caught in the act: in vivo mapping of macrophage infiltration in nerve injury by magnetic resonance imaging. J. Neurosci. $\mathbf{2 3}$. 10892-10896 (2003)

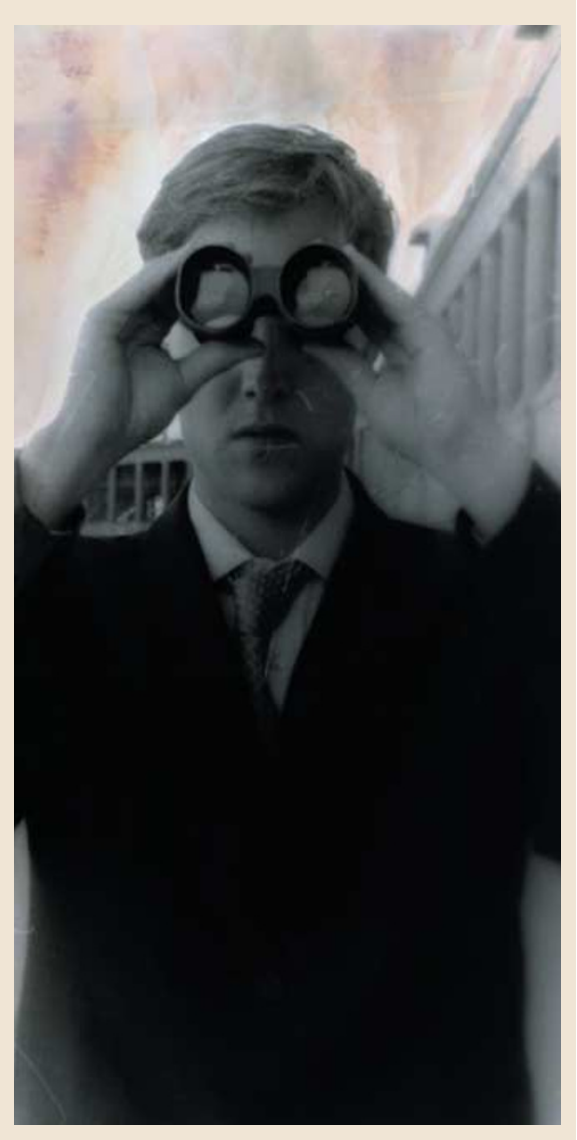

\section{AUTONOMIC NERVOUS SYSTEM}

\section{Circuit training}

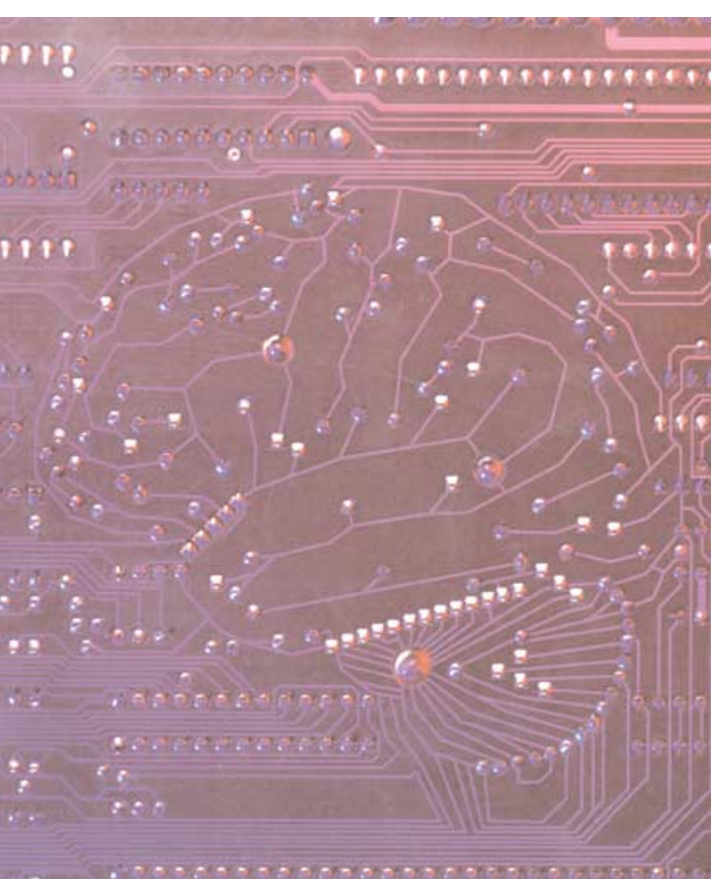

The visceral reflex circuits are responsible for modulating vital bodily functions, including breathing, digestion and heartbeat. New findings by Dauger et al., reported in Development, reveal a specific role for the homeodomain transcription factor Phox $2 b$ in the construction of these circuits.

The same team previously showed that the Phox $2 b$ gene is required for the development of the efferent (motor) arm of the visceral nervous system, and their new data indicate that the gene is also required for the development of afferent (sensory) pathways. In homozygous Phox $2 b$ knockout mice, the nucleus of the solitary tract (nTS) - a hindbrain nucleus that receives and relays sensory information from the visceral organs - fails to differentiate. In addition, sensory structures that project to the nTS, including sensory ganglia, the area postrema and the carotid body, are absent in mice that lack Phox $2 b$.

In humans, heterozygous mutations in $P H O X 2 B$ are associated with congenital central hypoventilation syndrome (CCHS), a childhood condition in which the autonomic control of breathing is impaired, particularly during the rapid eye movement phase of sleep. Dauger et al. showed that heterozygous Phox $2 b$-knockout mice also show defects in breathing control during early postnatal life. In particular, young mutant animals showed a reduced ventilatory response to raised levels of carbon dioxide (hypercapnia). So, the heterozygous Phox $2 b$ knockout seems to provide a good animal model for CCHS.

Phox $2 b$ is a rare example of a gene that controls the development of a specific circuit, which is constructed from different neuronal subtypes from diverse origins, including the neural tube, neural crest and epibranchial placodes. This poses an intriguing question: how does the developing embryo bring the Phox $2 b$ expressing cells into contact to make the appropriate synaptic connections? Dauger et al. suggest that Phox $2 \mathrm{~b}$ might regulate the expression of molecules that are required for axon guidance and/or neuronal migration, and an appropriately timed inactivation of Phox $2 b$ (to bypass its requirement for earlier stages of neuronal differentiation) could provide answers to this question.

Heather Wood

\section{Q) References and links}

ORIGINAL RESEARCH PAPER Dauger, S. et al. Phox $2 b$ controls the development of peripheral chemoreceptors and afferent visceral pathways. Development 130, 6635-6642 (2003) FURTHER READING Pattyn, A. et al. The homeobox gene Phox $2 b$ is essential for the development of autonomic neural crest derivatives. Nature 399, 366-370 (1999) | Pattyn, A. et al. Control of hindbrain motor neuron differentiation by the homeobox gene Phox2b. Development 127, 1349-1358 (2000) 